\title{
Osteonecrosis de los maxilares inducida por bifosfonatos: prevención y actitud terapéutica
}

\section{Bisphosphonate induced osteonecrosis of the jaws: prevention and therapeutic approach}

\author{
F.J. Barrientos Lezcano', B. Peral Cagigal'1, G. de la Peña Varela', L.A. Sánchez Cuéllar², \\ J.M. García Cantera², A. Serrat Soto², A. Verrier Hernández ${ }^{3}$
}

Resumen: Introducción. La osteonecrosis maxilar o mandibular por bifosfonatos puede convertirse en una epidemia debido a la amplia difusión de estos fármacos entre la población.

Material y método. Se muestra un protocolo para la prevención y el tratamiento de esta enfermedad. Se presentan tres casos de osteonecrosis maxilar/mandibular.

Resultados. Es difícil lograr una curación completa; sin embargo es posible detener la progresión de la enfermedad.

Discusión. La cirugía y la suspensión de la terapia con bifosfonatos han demostrado poca utilidad. Los antibióticos y los enjuagues con clorhexidina son las únicas medidas eficaces.

Conclusiones. Es imprescindible una planificación adecuada previa a la instauración del tratamiento con bifosfonatos. Ante una osteonecrosis establecida, la actitud debe ser conservadora.

Palabras clave: Bifosfonatos; Osteonecrosis; Necrosis avascular; Maxilar; Mandíbula.

Recibido: 24.04 .06

Aceptado: 18.12 .06

\begin{abstract}
Introduction. Bisphosphonate-induced osteonecrosis of the jaws might reach epidemic proportions due to the widespread use of this therapy.

Materials and methods. A protocol for prevention and treatment of this pathology is shown. Three clinical cases are reported.

Results. It is quite difficult to reach restitutio ad integrum, but stopping the progress of the disease is possible.

Discussion. Surgical treatment and cessation of bisphosphonate therapy are of no use. Only antibiotics and oral chlorhexidine have shown some benefits.

Conclusions. An accurate preventive attitude is mandatory prior to undergoing bisphosphonate therapy. If osteonecrosis of the jaws is present, management should be conservative.
\end{abstract}

Key words: Osteonecrosis; Bisphosphonates; Avascular necrosis; Jaws; Mandible.

\footnotetext{
1 Médico Residente.

2 Médico Adjunto.

3 Jefe de Servicio.

Servicio de Cirugía Oral y Maxilofacial.

Correspondencia:

Dr. F.J. Barrientos

Hospital Universitario Pio del Rio Hortega

c/ Torquemada s/n

47010 Valladolid, España

Email: barryentos@gmail.com
}

Hospital Universitario Pio del Río Hortega. Valladolid, España 


\section{Introducción}

La exposición de hueso necrótico maxilar o mandibular en pacientes tratados con bifosfonatos se está convirtiendo en un motivo de consulta cada vez más frecuente. Los bifosfonatos, ${ }^{1}$ (Tabla 1) son análogos no metabolizados del pirofosfato, con afinidad por el tejido óseo. Actúan sobre los osteoclastos (a través de un receptor de membrana o de una enzima intracelular), ${ }^{2}$ inhibiendo su quimiotaxis, acortando su vida media, frenando su actividad e induciendo su apoptosis. ${ }^{3}$ Como consecuencia, detienen la reabsorción ósea. El zoledronato y el pamidronato inhiben además la neoangiogénesis capilar tumoral.4-6

Generalmente, ${ }^{7}$ están indicados para estabilizar la pérdida de masa ósea en mujeres postmenopáusicas con osteoporosis (vía oral). La administración intravenosa queda reservada a casos de metástasis óseas (principalmente de mama y próstata) y para corregir la reabsorción ósea o la hipercalcemia moderada-severa en casos de mieloma múltiple. También se recomienda su uso en lesiones osteolíticas causadas por cualquier tumor sólido.2,8

Marx, 7 publicó en el año 2003 una serie de 36 casos de exposición ósea maxilar o mandibular por bifosfonatos. Desde entonces se han comunicado numerosos casos, y todavía cabe esperar muchos más dada la amplia difusión de estos fármacos entre la población.

La historia de la enfermedad, ${ }^{2,9}$ típicamente comienza con una alveolitis postexodoncia (aunque hay casos espontáneos) refractaria a tratamiento, que progresa hacia una osteomielitis, con secuestros óseos, exposición ósea, inflamación y supuración. Con frecuencia se realizan biopsias para descartar metástasis óseas mandibulares o tumores maxilares óseos primarios. Al intentar cubrir esas áreas se fracasa aunque se haya llevado a cabo una meticulosa regularización o resección ósea y cierre con colgajos mucosos locales. Clínica y radiológicamente las lesiones recuerdan a las de la osteoradionecrosis, con secuestros (formados espontáneamente o tras procedimientos invasivos) que pueden complicarse con infecciones secundarias. ${ }^{2}$

Su patogenia radica en la interrupción de la actividad remodeladora osteoclástica y del recambio (turnover) celular óseo, ${ }^{7}$ agravada por una insuficiencia vascular localizada como consecuencia de cambios isquémicos. ${ }^{2}$ Esta inhibición es parcial en el caso de bifosfonatos orales, produciéndose osteonecrosis raramente y sólo en casos de altas dosis acumuladas tras largos periodos de tiempo; si se administran vía intravenosa, la inhibición osteoclástica es irreversible, produciéndose su apoptosis.?

Esta entidad es característica del hueso maxilar o mandibular; estos fármacos tienen afinidad por él debido a su aporte vascular

\section{Introduction}

The exposure of necrotic maxillary 0 mandibular bone in patients treated with bisphosphonates is becoming increasingly questionable. Bisphosphonates' (Table 1) are non-metabolizable pyrophosphate analogs, with a preference for bone tissue. They act on osteoclasts (through a membrane receptor or an intracellular enzyme), ${ }^{2}$ inhibiting chemotaxis, shortening their average life span, halting their activity and inducing apoptosis. ${ }^{3}$ As a result, bone resorption is stopped. Zoledronate and pamidronate also inhibit capillary neoangiogenesis of the tumor. ${ }^{4-6}$

Generally ${ }^{7}$ bisphosphonates are indicated for stabilizing the loss of bone mass in postmenopausal women with osteoporosis (and they are taken orally). Intravenous administration is reserved for cases of bone metastases (principally breast and prostrate) and for correcting bone resorption, or moderate-to-severe hypercalcemia in cases of multiple myeloma. It is also recommended for osteolytic lesions caused by any type of solid tumor. ${ }^{2,8}$ In 2003 Marx 7 published a series of 36 maxillary or mandibular exposure cases due to bisphosphonates. From then on numerous cases have been reported, and many more are to be expected given the wide use of these drugs among the population.

The initial phase of the disease 2,9 typically starts with post-extraction alveolitis (although there are spontaneous cases) refractory to treatment, which progresses towards osteomyelitis, with sequestrum, bone exposure, inflammation and suppuration. Biopsies are frequently carried out in order to rule out mandibular bone metastases or primary jaw bone tumors. Failure occurs when trying to cover these areas even though meticulous regularization or bone resection has been carried out together with closure with local mucosal flaps. Clinically and radiologically the lesions are like those from osteoradionecrosis with sequestrum (that form spontaneously or after invasive procedures) that can complicate due to secondary infections. ${ }^{2}$

Its pathogenesis lies in the interruption of osteoclastic remodeling activity and from bone cell turnover, ${ }^{7}$ which is aggravated by localized vascular insufficiency as a result of 
profuso y a su elevado turnover celular (hay gran actividad de remodelación ósea alrededor del ligamento periodontal). Otros factores incluyen el escaso espesor de la mucosa maxilar-mandibular y la frecuente "agresión" a la que se ve sometido el hueso en forma de procedimientos quirúrgicos u odontológicos invasivos.

Predomina en maxilar superior (38$80,5 \%)$; el $14-63 \%$ se localiza en mandíbula y el 5,5-23\% en ambos.2,10

\section{Material y método}

\section{Caso clínico 1}

Paciente mujer de 37 años remitida (mayo del año 2005) a nuestro Servicio por dolor neuropático trigeminal derecho de año y medio de evolución que debutó 20 días tras la extracción incompleta del cordal inferior derecho realizada (año 2004) por su odontólogo. En sus antecedentes destacaba un cáncer de mama (año 2001), tratado con cirugía y quimioterapia. Posteriormente se le diagnosticaron metástasis óseas metacrónicas en columna vertebral lumbar (año 2002) y cerebrales (año 2003), ambas tratadas con radioterapia. Debido a la hipercalcemia causada por las metástasis óseas se instauró tratamiento con zoledronato (infusión de $4 \mathrm{mg} / \mathrm{semana}$ ) durante año y medio; se le suspendió durante seis meses y se reinstauró de nuevo. También recibió tratamiento con corticoides (pauta desconocida).

En la exploración oral se observaba supuración y gingivitis alrededor del resto radicular de 48. El diagnóstico diferencial se planteó entre una osteítis crónica/osteomielitis y una metástasis ósea. Clínica y radiológicamente (Fig. 1) afectaba también al 47, por lo que se realizó avulsión del mismo y legrado con ostectomía del lecho alveolar de 48 previa extracción del resto radicular (Fig. 2). La histopatología descartó infiltración neoplásica, observándose únicamente tejido de granulación que contenía en su espesor espículas óseas. Tres meses después persistía la exposición ósea; se realizó una regularización ósea y cierre del defecto con colgajo mucoso local, enviándose de

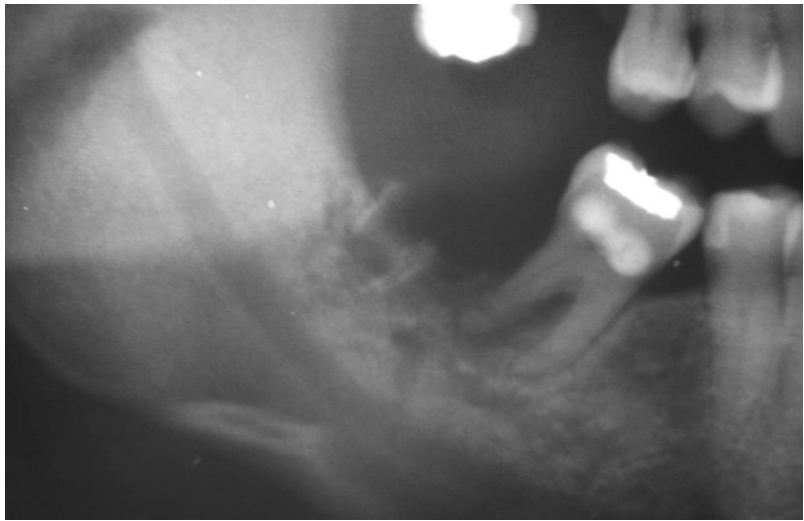

Figura 1. Imagen de radiografía panorámica que muestra el área patológica cuando la paciente fue remitida. Se aprecia el resto radicular del cordal inferior derecho y afectación periodontal de la pieza anterior.

Figure 1. Panoramic radiography showing pathological area on referral. The root remains of the lower right wisdom tooth can be appreciated and periodontal involvement of this tooth.

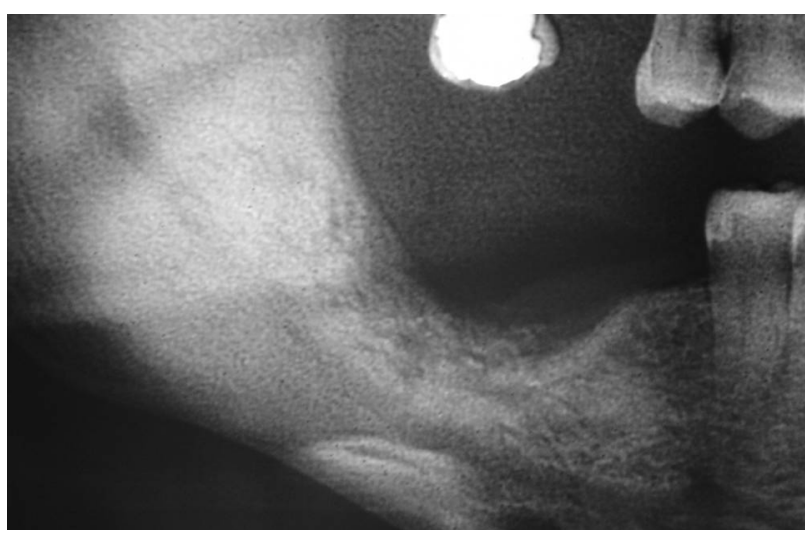

Figura 2. Detalle de la OPG tras la extracción de la pieza 47 y del resto radicular, con legrado del lecho óseo alveolar.

Figure 2. OPG detail after extraction of tooth 47 and root remains with curettage of the alveolar bony bed.

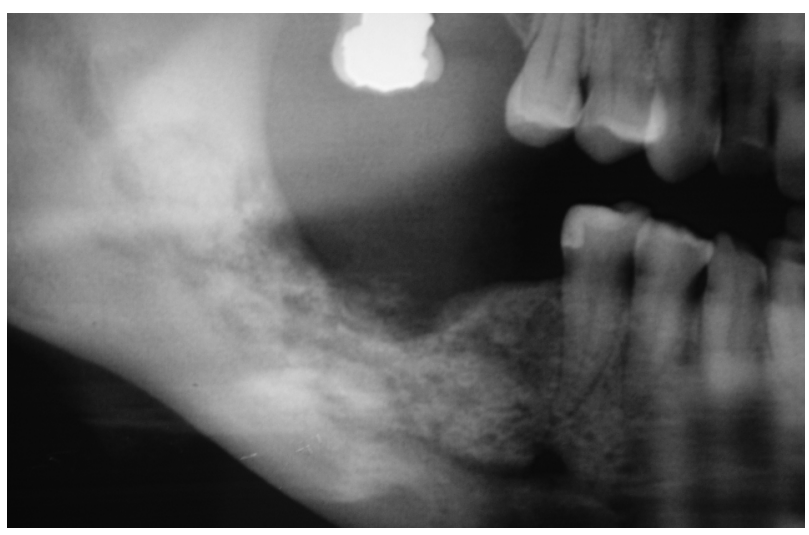

Figura 3. Detalle de la OPG mostrando la persistencia y avance de la osteonecrosis 9 meses después de las extracciones y regularización ósea.

Figure 3. OPG detail showing persistence and advancement of osteonecrosis 9 months after the extractions and bone regularization. ischemic changes. ${ }^{2}$ This inhibition is partial in the case of oral bisphosphonates, as osteonecrosis rarely arises and only following high accumulated doses over long periods of time. If administered intravenously, osteoclastic inhibition is irreversible, and apoptosis will take place.

This is a characteristic entity of the jaw bones. These drugs have a preference for them given their profuse vascular supply and the high cellular turnover (there is great bone remodeling activity around the periodontal ligament). Other factors include the limited thickness of the mandibular-maxillary mucosa and the frequent "aggressive behavior" suffered by the bone in the way of invasive surgical or dental procedures. It predominates in the upper jaw (38-80.5\%); while 14$63 \%$ is located in the mandible and $5.5-23 \%$ in both. 2,10

\section{Material and method}

\section{Case report 1}

Female patient, 37 years old, was referred (May 2005) to our department because of right-sided trigeminal neuropathic pain that had been evolving for a year and a half, and which had arisen 20 days after the incomplete extraction of the lower right wisdom tooth carried out (in 2004) by her dentist. Her medical history included breast cancer (2001) that had been treated with surgery and chemotherapy. Metachronic bone metastases was then diagnosed in the lumbar spine (in 2002) and brain (2003), both treated with radiotherapy. Due to hypercalcemia caused by the bone metastases, treatment 
nuevo una muestra de tejido óseo para su análisis, que determinó la presencia de colonias bacilares de tipo actinomycótico rodeando fragmentos compactos de hueso e infiltrando sus espacios medulares. Se prescribió tratamiento inicial con amoxicilina- clavulánico 875/125 /8 h durante 2 semanas y mantenimiento con amoxicilina 500mg/8h sin interrupción hasta la fecha. En revisiones bimensuales posteriores la lesión conserva el mismo aspecto clínico, aunque radiológicamente se observa un avance de la rarefacción ósea hacia sínfisis (Fig. 3). La paciente refiere ocasionales episodios autolimitados de tumefacción gingivo-vestibular. La sintomatología ha cedido considerablemente y sólo refiere leves molestias en los citados episodios de sobreinfección.

\section{Caso clínico 2}

Paciente mujer de 68 años con antecedente de mieloma múltiple lgG estadio III B (año 2003) tratado con quimioterapia (6 ciclos de VBMCP / VBAD), con respuesta completa. Posteriormente (año 2004) se le realizó autotransplante de progenitores hemopoyéticos de sangre periférica. Presentaba múltiples aplastamientos vertebrales y lesiones focales óseas secundarias al mieloma por lo cual recibía tratamiento con alendronato (10 mg/24 h, vía oral) sin interrupción, prednisona (50 mg/2 días, vía oral) y varios analgésicos. Entre diciembre del año 2004 y febrero del 2005 su odontólogo le realizó exodoncia de todas las piezas dentales (incisivos, caninos y premolares superiores e inferiores) tras lo cual ha sido portadora de prótesis total removible. Fue remitida (julio del 2005) por presentar áreas de exposición ósea con necrosis en los cuadrantes 1,2 y 3 (Figs. 4 y 5) y molestias a nivel local. Ante la sospecha clínica y radiológica (Fig. 6) de osteomielitis se le aconsejó prescindir temporalmente de la prótesis, se le pautó tratamiento con clindamicina 300 mg / 8h / 7 días y se le hizo secuestrectomía, regularización ósea y cierre directo del defecto, previo despegamiento mucoperióstico vestibular y palatino. Se logró cubrir por completo el hueso expuesto. No se



Figura 4. Exposición de hueso necrótico mandibular en el área en que se realizó la extracción.

Figure 4. Exposure of necrotic mandibular bone in extraction area.

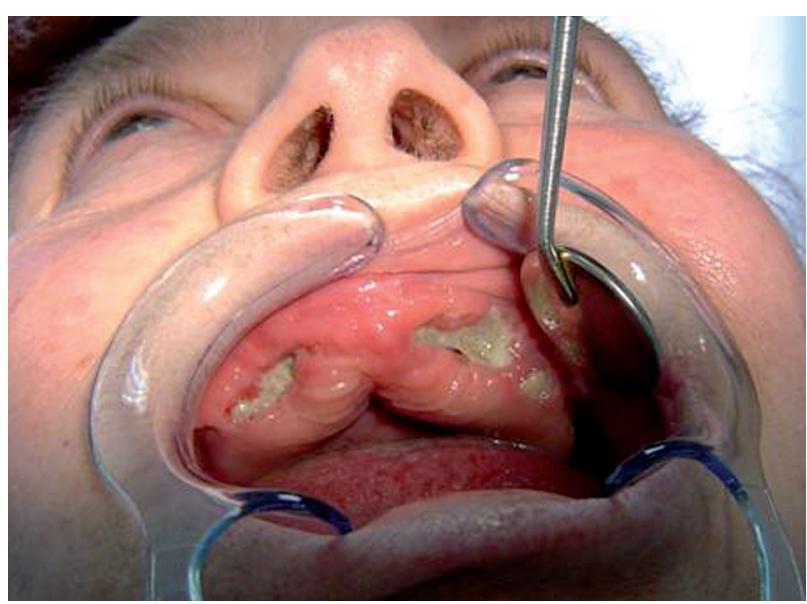

Figura 5. Osteonecrosis maxilar bilateral post-exodoncia. Figure 5. Bilateral maxillary osteonecrosis after extractions.

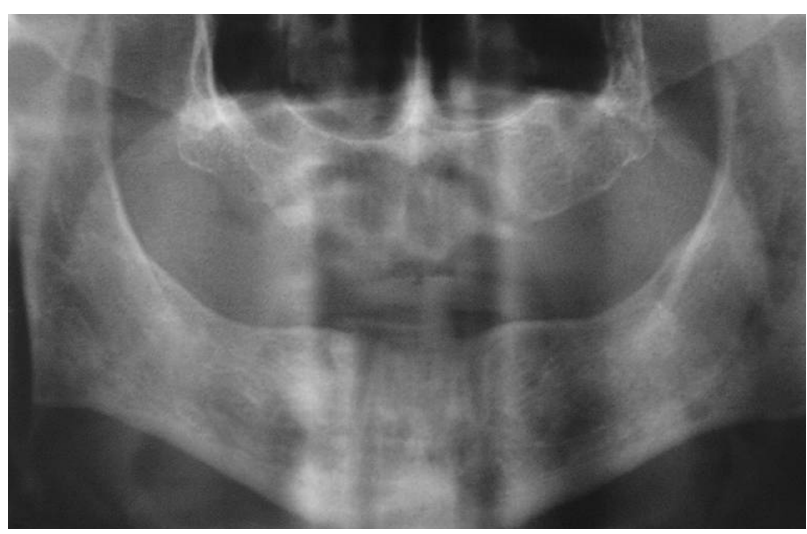

Figura 6. OPG de la paciente, mostrando áreas de irregularidad ósea alveolar a nivel bimaxilar, donde fueron practicadas las exodoncias.

Figure 6. OPG of patient showing areas with bimaxillary alveolar bone irregularity where extractions were carried out. with zoledronate was given (4mg infused per week) for a year and a half. This was suspended for six months and then started again. She was also given corticoid steroids (unknown dosage).

The oral examination revealed suppuration and gingivitis around the root remains of tooth 48. The differential diagnosis included chronic osteitis/osteomyelitis and bone metastasis. Tooth 47 was affected clinically and radiologically and avulsion was carried out together with curettage and an ostectomy of the alveolar bed of tooth 48 before extraction of the root remains (Fig. 2). The histopathologic report ruled out neoplastic infiltration, and only granulation tissue was observed that contained bone spicules. Three months later bone exposure persisted. Bone regulation was carried out and the defect was closed using a local mucosal flap, and a bone tissue sample was once again sent away for analysis that indicated the presence of bacillary colonies of an actinomycotic type surrounded by compacted bone fragments, which were infiltrating the medullary areas. Initial treatment was prescribed with amoxicillin-clavulanate $875 / 125$ every 8 hours for two weeks that was maintained with continuous amoxicillin 500 mg every 8 hours to date. The lesion was re-examined every two months and it continued to have the same clinical appearance, although radiologically bone rarefaction could be observed towards the symphysis (Fig. 3). The patient reported occasional self-limited bouts of vestibular-gingival swelling. The symptoms ceased considerably and she now only complains of slight discomfort from these bouts of infection. 
envió material para su estudio histológico. Un mes después y en sucesivas revisiones posteriores, las áreas de osteonecrosis han aparecido en la misma localización. Al sospechar osteonecrosis por bifosfonatos se le pautó amoxicilina- clavulánico 875/125 cada 8 horas durante 1 mes y enjuagues orales cada 12 horas con clorhexidina al $0,12 \%$. Se aconsejó demorar el uso de la prótesis. No se realizó biopsia ósea para evitar la progresión de las lesiones. Durante su seguimiento previo (bimensual) las lesiones no remitieron, aunque tampoco empeoraron. La paciente manifestó una mejoría subjetiva en la sintomatología. Falleció recientemente por un infarto cerebral debido a una estenosis carotídea.

\section{Caso clínico 3}

Paciente mujer de 81 años de edad, con esquizofrenia y graves síntomas extrapiramidales secundarios al tratamiento de la misma, remitida (mayo del año 2005) por el geriatra de su residencia por exposición ósea maxilar izquierda de $4-5 \mathrm{~cm}$ (Fig. 7). Edéntula no portadora de prótesis. El tiempo de evolución, clínica subjetiva y posibles factores precipitantes se desconocen al no contar con la colaboración de la paciente. Antecedente en el año 1998 de mieloma múltiple (IgG kappa) refractario en progresión (actualmente estadio III-B), con osteopenia de huesos largos y espondiloartrosis dorsolumbar, recibiendo desde entonces infusiones intravenosas de ácido zoledrónico (4 mg/mes). Aunque se sospechó osteonecrosis maxilar por bifosfonatos, se biopsió un pequeño fragmento óseo para descartar una metástasis ósea. La histopatología descartó neoplasia y fue informada como hueso necrótico con numerosas células inflamatorias y colonias de bacilos de tipo actinomyces. Se instauró tratamiento con amoxicilina-clavulánico (875/125, cada 8 horas, 15 días), metronidazol (500 mg/ 8 h. vía oral, 15 días) y enjuagues de clorhexidina al $0,12 \%$ cada 12 horas. Posteriormente recibió amoxicilina $500 \mathrm{mg} / 8 \mathrm{~h}$ durante 3 meses. Se recomendó a sus cuidadores el mantenimiento de una higiene oral estricta.

En revisiones posteriores realizadas con una periodicidad trimestral no se ha observado mejoría en el aspecto clínico de la lesión, aunque su progresión se ha detenido. No ha vuelto a recibir antibióticos. corto/medio plazo



Figura 7. Extensa exposición ósea maxilar izquierda. Figure 7. Extensive bone exposure on left side of maxilla.

Prevención en pacientes que van a recibir bifosfonatos (especialmente i.v)

- Objetivo: evitar infecciones y procedimientos dentales invasivos a

- Exploración dental incluyendo OPG \pm periapicales; descartar infecciones.

- Inspección oral por el odontólogo antes del tratamiento y en el seguimiento.

- Educar al paciente en la importancia de una buena higiene oral y en la notificación de síntomas.

- Examen de prótesis dentales removibles, confirmar su ajuste y retirarla por la noche.

- Profilaxis dental, control de caries y cuidado dental fortificante.

- Profilaxis antibiótica sólo para procedimientos invasivos (Exodoncia, Endodoncia, Periodoncia) y demorar tratamiento con bifosfonatos 1 mes.

- Penicilina.

- Alérgico: Quinolona o Eritromicina + Metronidazol.

- Dientes recuperables: rehabilitación funcional, incluso endodoncia.

- Dientes irrecuperables o con absceso: exodoncia y extirpación del tejido periodontal afectado.

- Seguimiento: revisiones periódicas cada 3-4 meses.

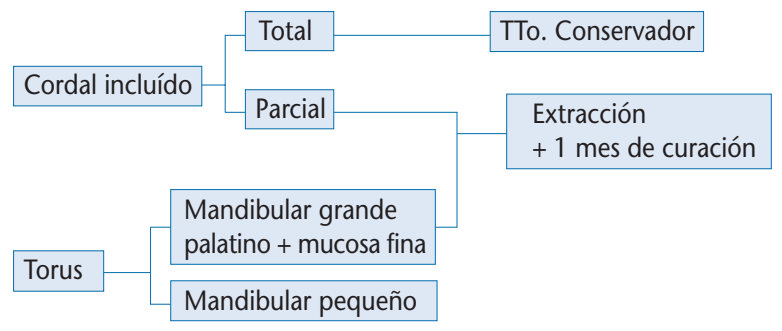

Figura 8. Recomendaciones previas a la instauración de una terapia prolongada con bifosfonatos. 7,11

Figure 8. Recommendations before commencing prolonged bisphosphonate therapy.
Case report 2

Female patient, 68 years old with a history of multiple myeloma IgC stage III B (in 2003) who had been treated with chemotherapy (6 cycles of VBMCP/VBAD) with complete response. Later (in 2004) an autotransplant was carried out with hemopoietic progenitors from peripheral blood. She had multiple crushed vertebrae and focal bone lesions secondary to the myeloma and, as a result, she was being treated with constant alendronate $(10 \mathrm{mg}$ taken orally every 24 hours), prednisone (50 mg every 2 days taken orally) and various analgesics. Between December 2004 and February 2005 all her teeth were extracted by her dentist (incisors, canines and upper and lower premolars) after which she was fitted with a removable complete prosthesis. She was referred to our department (in July 2005) because of bone exposure with necrosis in areas 1,2 and 3 (Figs. 4 and 5) and local discomfort. As there was a clinical and radiological suspicion (Fig. 6) of osteomyelitis, she was advised not to use the prosthesis temporarily. She was treated with clindamycin, 300mg every eight hours for a week, and a sequestrectomy was carried out together with bone regularization and direct closure of the defect, prior to raising a mucoperiosteal flap from the vestibular and palate side. The exposed bone was completely covered. No material was sent away to be studied histologically. A month later, during re-examination, the osteonecrotic areas appeared in the same location. As osteonecrosis was suspected due to bisphosphonates, 875/125 amoxicillin-clavulanate was administered every eight hours for a month, and oral rinses every 12 hours with chlorhexidine at $0.12 \%$. She was told to delay using the prosthesis. A bone biop- 


\section{Discusión}

Es indiscutible el valor de los bifosfonatos en pacientes oncológicos con metástasis óseas; aumentan la supervivencia y reducen las complicaciones esqueléticas y el dolor asociado a las mismas, mejorando su calidad de vida. ${ }^{2}$

Esta "epidemia" de osteonecrosis maxilar o mandibular por bifosfonatos ha generado confusión debido a la dificultad para curar por completo la enfermedad. En el año 2004 un panel de expertos en la enfermedad, ${ }^{11}$ se reunieron ante la creciente incidencia de casos y publicaron una serie de recomendaciones (Figs. 8 y 9) (Tabla 2).

A pesar de estas recomendaciones, el tratamiento óptimo de la osteonecrosis sigue siendo controvertido. Una de las pocas medidas efectivas es el tratamiento antibiótico aunque no esté bien definida la duración ideal del mismo. Se ha propuesto, ${ }^{10}$ su uso en forma de ciclos intermitentes o contínuos para evitar la osteomielitis y prevenir sobreinfecciones en partes blandas. Clínicamente son eficaces controlando el dolor y la evolución de la enfermedad.7,10 Esto se ha comprobado en los tres casos que presentamos; desde que se instauraron los antibióticos la sintomatología mejoró y la exposición ósea no progresó. Aunque se ha recomendado, 10 la clindamicina por su eficacia contra flora Gram- y por su afinidad hacia tejidos óseos, 10,11 Marx la desaconseja como terapia única, 7 y recomienda derivados de la penicilina como fármacos de primera elección. El antibiótico administrado debe cubrir géneros del tipo Eikenella o Actinomyces, gérmenes que con frecuencia colonizan (como ocurrió en los dos casos clínicos en los que se hizo biopsia ósea) el hueso expuesto. 7,10 También aconsejan procedimientos mínimamente invasivos como irrigaciones y lavados con antisépticos. $2,7,10$

Se advierte contra la realización de maniobras quirúrgicas como desbridamientos de heridas, decorticaciones óseas o extracciones dentarias. ${ }^{10}$ En efecto, no parece sensato hacer exodoncias cuando éste es precisamente el desencadenante típico de la enfermedad (casos 1 y 2); además, en ambos casos se ha visto cómo el legrado y regularización ósea han fracasado y las lesiones han recidivado. El primer caso incluso empeoró hasta que se adoptó una actitud conservadora, sin duda porque en el momento inicial no se consideró la posibilidad de que los bifosfonatos fueran causantes de la lesión. Por lo tanto no se debe descuidar la realización de una meticulosa historia clínica y anotar los fármacos que toma el paciente, entre los cuales se encuentra con frecuencia "una pastilla que toma para los huesos" a la que habitualmente no se le da importancia.

Hay casos en que los antibióticos fracasan, posiblemente debido a la dificultad de alcanzar una diana sometida a un entorno isquémico. En estos casos, si son leves, algunos autores, 10 aconsejan hacer sy was not carried out in order to avoid any progression of the bone lesions. During previous monitoring (bi-monthly) the lesions showed no sign of remission, although they had not become any worse. The patient's symptoms showed subjective improvement. She died recently due to a stroke secondary to carotid stenosis.

\section{Case report 3}

Female patient, 81 years old with schizophrenia and serious extrapyramidal symptoms secondary to her treatment, was referred to us (in May 2005) by the geriatrician of her residence as a result of maxillary bone exposure on the left side of 4-5 $\mathrm{cm}$ (Fig. 7). She was edentulous and did not have a prosthesis. As there was no patient collaboration, the time that had evolved, the subjective clinical data and possible triggering factors, could not be ascertained. She had a history of refractory multiple myeloma (Kappa lgG) in 1998 with evidence of progression (currently stage III-B) with long bone osteopenia and lumbar-back spondyloarthrosis and she had received intravenous infusions of zoledronic acid (4mg/month). Although maxillary osteonecrosis was suspected as a result of bisphosphonates, a biopsy was taken of a small bone fragment so that bone metastasis could be ruled out. The histopathological report ruled out any neoplasm and necrotic bone was reported with numerous inflammatory cells and colonies of the actinomyce-type bacillus. She was treated with amoxicillin clavulanate (875/125, every 8 hours for 2 weeks) metronidazole (500 mg taken orally every 8 hours for two weeks) and $0.12 \%$ chlorhexidine washes every 12 hours. She later received amoxicillin 500mg every 8 hours for 3 months. Her carers were advised to maintain strict oral hygiene. She was then re-examined every three months, but an improvement was not observed in the clinical appearance of the lesions although their growth had been halted. She did not receive any further antibiotic treatment.

\section{Discussion}

Bisphosphonates are of undoubted value for oncological patients with bone metastases. Survival increases, skeletal complications and associated pain are reduced, and quality of life is improved. ${ }^{2}$

This "epidemic" of maxillary or mandibular osteonecrosis as a result of bisphosphonates, has generated confu- 
desbridamiento óseo y cierre con colgajos mucosos, siempre bajo cobertura antibiótica. Esta actitud fracasó en los casos 1 y 2 a pesar de haber cerrado totalmente el defecto con colgajos mucoperiósticos. Marx ya advertía, ${ }^{13}$ de la inutilidad de este tipo de colgajos.

Hay casos severos en los que la magnitud del área expuesta de hueso o la sintomatología obligan a realizar resecciones totales o subtotales del hueso afectado y reconstrucción secundaria usando tejidos viables. ${ }^{10}$ Sin embargo, esta actitud incrementa el riesgo de sufrir fracturas patológicas así como nuevas áreas de osteonecrosis. ${ }^{10} \mathrm{En}$ el caso 3 casi la mitad del maxilar está expuesta; afortunadamente se sospechó desde el principio la implicación del ácido zoledrónico (también presente en el caso 1) y se adoptó una conducta conservadora; además el estado general de la paciente y su corta esperanza de vida desaconsejaban una intervención quirúrgica. Ruggiero y cols ${ }^{2}$ realizan secuestrectomías sistemáticas de los fragmentos óseos que no responden a tratamiento conservador, ya que constituyen un posible foco de sobreinfecciones; el problema en estos casos es encontrar un margen quirúrgico viable de hueso ya que los bifosfonatos están depositados en todo el tejido óseo y no sólo en el hueso necrótico expuesto, siendo probable una ulterior exposición del margen de resección. 2,9

En pacientes sintomáticos con fracturas patológicas se podría intentar una resección mandibular segmentaria $y$ reconstrucción con placa rígida, ${ }^{2}$ quedando formalmente contraindicados los colgajos libres o los injertos vascularizados por la posibilidad de desarrollar necrosis en los márgenes. ${ }^{2}$
Tabla 2. Tratamiento de la osteonecrosis por bifosfonatos, 71

\section{Tratamiento de la osteonecrosis establecida}

- Consulta con cirujano maxilofacial y odontólogo; seguimiento cada 3 meses (antes si empeoramiento).

Prevención de lesiones óseas adicionales

- Regularización mínima de bordes óseos agudos traumáticos sobre mucosa.

- Prótesis / tope removible (acrílico o vinilo) protectora del hueso expuesto.

- No biopsia (excepto sospecha de metástasis).

- No implantes osteointegrados.

Tratamiento antibiótico

- Cursos intermitentes o contínuos.

- Cultivos rutinarios (considerar aerobios, anaerobios, virus y hongos).

- Enjuagues orales de gluconato de clorhexidina 0,12\%.

- Bolsas periodontales de clorhidrato de minociclina.

Tratamiento estándar

Amoxicilina $500 \mathrm{mg} / 6 \mathrm{~h}+$ Clorhexidina $0,12 \%$

Refractario o muy sintomático

añadir Metronidazol $500 \mathrm{mg} / 8 \mathrm{~h}$ vo.

Celulitis severa / paciente ingresado

Amoxicilina-Clavulánico 1000/200 /6 h iv + Metronidazol 500 mg/8 h iv

Alérgico

Ciprofloxacino 500 mg/12 h ó Eritomicina $400 / 8$ h vo. + Metronidazol

$500 \mathrm{mg} / 8 \mathrm{~h}$ vo.

Suspensión/interrupción bifosfonatos?

- Valorar riesgo osteonecrosis vs. riesgo suspensión tratamiento.

- Siempre en coordinación con oncólogo.

Table 2. Osteonecrosis treatment with bisphosphonates 7,11

Treatment for established osteonecrosis

- Consultation with maxillofacial surgeon and dentist: follow-up every 3 months (or before if patient worsens)

Prevention of additional bone lesions

- Minimal regularization of traumatic sharp bone edges affecting mucosa.

- Removable prosthesis/ splint (acrylic or vinyl) to protect exposed bone.

- No biopsies (except when metastasis is suspected).

- No osseointegrated implants.

Antibiotic treatment

- Intermittent or continuous antibiotic course.

- Routine cultures (aerobic, anaerobic, virus and fungi should be taken into consideration).

- Oral rinses with chlorhexidine gluconate $0.12 \%$.

- Periodontal sacs of minocycline chlorhydrate.

Standard treatment

Amoxycillin $500 \mathrm{mg} / 6 \mathrm{~h}+$ Chlorhexidine $0.12 \%$

Refractory or with considerable symtomatology add Metronidazole $500 \mathrm{mg} / 8 \mathrm{~h}$ orally.

Severe cellulits/admitted patient

Amoxycillin-Clavulanate 1000/200 /6 h iv + Metronidazole 500 mg/8 h iv

Allergy

Ciprofloxacin 500 mg/12 h or Erithomycin 400/8 h orally + Metronidazole $500 \mathrm{mg} / 8 \mathrm{~h}$ orally.

Suspension / interruption of bisphosphonates

- Evaluate risk of osteonecrosis vs. risk of suspending treatment

- There should always be coordination with the oncologist sion due to the difficulty for completely curing the disease. In 2004 a panel of experts on the disease ${ }^{11}$ gathered together because of the growing incidence of cases and a series of recommendations was published (Figs. 8 and 9) (Table 2).

In spite of these recommendations, the best treatment for osteonecrosis continues being controversial. One of the few effective measures is antibiotic treatment although the ideal duration has not yet been defined. Its use has been proposed ${ }^{10}$ in intermittent or continuous cycles so that osteomyelitis is avoided and further soft tissue infection is prevented. They are clinically efficient for controlling pain and the development of the disease. 7,10 This has been corroborated in the three cases presented. The symptoms improved as soon as the antibiotics were administered, and there was no increase in bone exposure. Although clindamycin has been recommended given its efficiency against Gram flora and because of its bone tissue preference, 10,11 Marx does not advise its use on its own, ${ }^{7}$ and he recommends penicillin derivatives such as first choice drugs. The antibiotic administered should cover the Eikenella or Actinomyces-type germs that 
En resumen, el tratamiento quirúrgico sólo debe considerarse en casos sintomáticos en los que fracasa el tratamiento conservador, ${ }^{2}$ y debe limitarse a secuestrectomías a demanda, ${ }^{9}$ retirando el menor hueso posible y preservando el periostio para mantener el hueso alejado del contacto directo con la flora oral. ${ }^{7}$

Otra medida que suscita controversia es la suspensión de la terapia con bifosfonatos (con el riesgo que conlleva para el paciente oncológico). Esta decisión, en caso de tomarse, debe hacerse en coordinación con el oncólogo.11,14 En ninguno de los tres casos clínicos se contempló esta posibilidad ya que todas tenían antecedentes neoplásicos y, las dos últimas, lesiones óseas cuya clínica estaba relativamente controlada con los bifosfonatos. Además, no se ha demostrado que retirar los bifosfonatos aporte algún beneficio una vez establecida la osteonecrosis, ${ }^{10}$ e incluso se ha descrito la aparición de nuevas áreas de necrosis tras la suspensión de la terapia. ${ }^{2}$ Se podría valorar si la indicación no fue oncológica, ${ }^{7}$ pero se desaconseja suspenderlos si fueron prescritos por dolor debido a metástasis óseas, fracturas patológicas o hipercalcemia inducida por un tumor. Sin embargo, el empeoramiento sobre la calidad de vida que en ocasiones produce la osteonecrosis supera ampliamente la mejoría en la misma atribuída a los bifosfonatos. ${ }^{2}$

Se ha propuesto la suspensión temporal en pacientes que se van a someter a intervenciones de cirugía oral; esta medida es inútil ya que los bifosfonatos se acumulan en la matriz ósea mineralizada (en relación directa con la dosis total acumulada y con la duración del tratamiento) ${ }^{10}$ y persisten en ella durante largos períodos de tiempo, ${ }^{10,15}$ siendo gradualmente reincorporados a la circulación a lo largo de meses y años. ${ }^{5}$ En estos casos es preferible realizar cultivos de la zona de extracción en el momento de la intervención y administrar antibióticos durante al menos 10 días. ${ }^{7}$

El oxígeno hiperbárico, a diferencia de la osteoradionecrosis, no ha demostrado una eficacia uniforme limitando el progreso de la enfermedad. ${ }^{2}$

En cuanto a los implantes, están contraindicados, si bien podría pensarse que al inhibir la reabsorción ósea los bifosfonatos deberían favorecer la osteointegración. Se han comunicado fracasos en pacientes con osteoporosis que tomaban bifosfonatos orales. ${ }^{2}$ Starck, ${ }^{16}$ comunicó un caso de fracaso repetido de rehabilitación implantológica debida al etidronato. Además, al carecer de "anclaje epitelial" los implantes predisponen a una exposición ósea posterior. ${ }^{7}$

\section{Conclusiones}

Se debe considerar a todo paciente tratado con bifosfonatos (especialmente intravenosos) como susceptible de presentar osteonecrosis en caso de someterse a procedimientos odontológicos invasivos o a cirugía ósea maxilar o mandibular. La clave es realizar una adecuada planificación antes de comenzar el tratamiento con bifosfonatos, de modo similar a los pacientes que van a someterse a radioterapia de cabeza y cuello. ${ }^{2}$ Es necesario anticiparse a los problemas odontológicos que podría tener el paciente a corto-medio plazo, solucionándolos con suficiente antelación para que, una vez instaurado el tratamiento con bifosfonatos, la mucosa se encuen- frequently colonize exposed bone (as occurred in both clinical cases in which a bone biopsy was carried out). ${ }^{7-10}$ They also advise minimally invasive procedures such as irrigation and antiseptic washes. $2,7,10$

Surgical procedures such as wound debridement are not advised nor are bone decortication or dental extraction. ${ }^{10}$ Carrying out extractions therefore does not appear to be very sensible, as this is precisely what typically triggers the disease (Cases 1 and 2). Both cases show how curettage and bone regularization failed and how the lesions recurred. The first case even worsened until a conservative approach was adopted, no doubt because initially the possibility of bisphosphonates being the cause of the lesion was not taken into account. Therefore carrying out a meticulous medical history should be stressed as should be making a note of the drugs being taken by the patient, together with the " tablet for the bones" which normally is not given much importance. There are cases in which antibiotics fail, possibly due to the difficulty of reaching a target subjected to ischemic surroundings. In these cases, if slight, some authors ${ }^{10}$ advise carrying out debridement and flap closure, but always with antibiotic cover. This approach failed in cases 1 and 2 in spite of completely closing the defect with mucoperiosteal flaps. Marx had already warned that these flaps were of little use. ${ }^{13}$

There are severe cases in which the magnitude of the exposed area of bone or the symptoms makes a total or subtotal resection necessary and secondary reconstruction using viable tissue. ${ }^{10}$ However, this approach increases the risk of experiencing pathological fractures as well as new area of osteonecrosis. ${ }^{10}$ In case $\mathrm{N}^{\circ} 3$, nearly half the maxilla was exposed. Fortunately, it was suspected from the start that Zoledronic acid was involved (also in case 1) and a conservative approach was adopted. In addition, the general state of the patient and her short life expectancy obviated surgical intervention. Ruggiero et al. ${ }^{2}$ carried out systematic sequestrectomies of the bone fragments that had not responded to conservative treatment, as they constituted a possible focus of additional infection. The problem in these cases is finding a viable surgical margin of bone, as bisphosphonates are deposited in all bone tissue, and not only in exposed necrotic bone, with ulterior exposure of the resection margin being possible.2,9

In symptomatic patients with pathologic fractures, segmental mandibular resection and reconstruction with a rigid plate 2 could be attempted. Free flaps or vascularized grafts are strictly contraindicated given the possibility of developing necrosis in the margins. ${ }^{2}$

In short, surgical treatment should only be considered in symptomatic cases in which conservative treatment has failed, ${ }^{2}$ and it should be limited to sequestrectomies when needed. ${ }^{9}$ The least amount of bone as possible should be removed and the periosteum should be preserved so that the bone does not come into contact with oral flora. ${ }^{7}$

Another step that has led to certain controversy is suspending bisphosphonate therapy (with the risk that this 
tre íntegra y no exista ningún área de exposición ósea.

Una vez establecida la osteonecrosis, la conducta debe ser conservadora ante la posibilidad de un empeoramiento yatrogénico del cuadro. Los procedimientos quirúrgicos, también conservadores, se reservarán para los casos más sintomáticos.

\section{Bibliografía}

1. Consejo General de Colegios Oficiales de Farmacéuticos. Catálogo de Medicamentos. Madrid: EINSA 2006.

2. Ruggiero S, Mehrotra B, Rosenberg T, Engroff S. Osteonecrosis of the jaws associated with the use of bisphosphonates: a review of 63 cases. J Oral Maxillofac Surg 2004;62:527-34.

3. Sarathy A, Bourgeois S, Goodell G. Bisphosphonate- associated osteonecrosis of the jaws and endodontic treatment: two case reports. J Endod 2005;31: 75963.

4. Fournier P, Boissier S, Filleur S, Guglielmi J, Colombel M. Bisphosphonates inhibit angiogenesis in vitro and testosterone-stimulated vascular regrowth in the ventral prostate in castrated rats. Cancer Res 2002;62:6538-44.

5. Lin JH, Russel RG, Gertz B. Pharmacokineticks of alendronate: an overview. Int J Clin Pract 1999;101:18-26.

6. Wood J, Bonjean K, Ruetz S, Bellahcène A, Devy L, Foidart J, y cols. Novel antiangiogenic effects of the bisphosphonate compound zoledronic acid. J Pharm Exp Ther 2002;302:1055-61.

7. Marx R, Sawatari Y, Fortin M, Broumand V. Bisphosphonate-induced exposed bone (osteonecrosis / osteopetrosis) of the jaws: risk factors, recognition, prevention and treatment. J Oral Maxillofac Surg 2005;63:1567- 75.

8. Melo M, Obeid G. Osteonecrosis of the maxilla in a patient with history of bisphosphonate therapy. J Can Dent Assoc 2005;71:111-3.

9. Hellstein J, Marek C. Bisphosphonate osteochemonecrosis (Bis-phossy jaw): is this phossy jaw of the 21st century? J Oral Maxillofac Surg 2005;63:682-9.

10. Lenz J, Steiner-Krammer B, Schmidt W, Fietkau R, Mueller P, Gundlach K. Does avascular necrosis of the jaws in cancer patients only occur following tratment with bisphosphonates? J Craniomaxillofac Surg 2005;33:395-403.

11. Damato K, Gralow J, Hoff A, Huryn J, Marx R, Ruggiero S, y cols. ADA council on scientific affairs. Expert panel recommendations for the prevention, diagnosis and treatment of osteonecrosis of the jaws: June 2004. (www.ada.org/prof/ resources/topics/topics_osteonecrosis_whitepaper.pdf).

12. Katz $\mathrm{H}$. Endodontic implications of bisphosphonate- associated osteonecrosis of the jaws: a report of three cases. J Endod 2005;31:831-4.

13. Marx R. Pamidronate (Aredia) and Zoledronate (Zometa) induced avascular necrosis of the jaws: a growing epidemic. J Oral Maxillofac Surg 2003;61:1115- 7.

14. Ficarra G, Beninati F, Rubino I, Vannucchi A, Longo G, Tonelli P, y cols. Osteonecrosis of the jaws in periodontal patients with a history of bisphosphonates treatment. J Clin Periodontol 2005;32:1123-8.

15. Rodan GA, Fleisch HA. Bisphosphonates: mechanisms of action. J Clin Invest 1996;97:2692-6.

16.- Starck WJ, Epker BN. Failure of osseointegrated dental implants after diphosphonate therapy for osteoporosis: a case report. Int J Oral Maxillofac Implants 1995; 10:74- 8. entails for the oncological patient). This decision, if taken, should be made together with the oncologist. ${ }^{11,14}$ In none of the three clinical cases was this possibility contemplated as they all had neoplastic antecedents and, the last two had bone lesions with a clinical course that was being relatively controlled with bisphosphonates. Moreover, it has not been demonstrated that there is any benefit to be had from stopping bisphosphonates once the osteonecrosis has started. ${ }^{10}$ This could be evaluated if the indication were not oncological, 7 but suspension is not advisable if being taken because of pain due to bone metastasis, pathological fractures or tumor-induced hypercalcemia. However, the worsening in quality of life that is sometimes produced by osteonecrosis is much greater than the improvement attributed to bisphosphonates. ${ }^{2}$

Temporally suspending treatment has been proposed for patients that are going to undergo oral surgery. These measures are of no use, as bisphosphonates accumulate in the mineralized bone matrix (and they are directly related to the total accumulated dosage and treatment duration $)^{10}$ and they remain here for long time periods, 10,15 gradually being reincorporated into the circulation over months and years. ${ }^{5}$ In these cases carrying out cultures in the extraction area at the time of the intervention and administering antibiotics for at least 10 days is preferable.

Hyperbaric oxygen, unlike with osteoradionecrosis, has not shown any uniform efficiency with regard to halting the progress of the disease. ${ }^{2}$

Implants are contraindicated, although one might think that if bone resorption is inhibited, the bisphosphonates will favor osseointegration. Failures have been reported in osteoporosis patients who were taking bisphosphonates orally. ${ }^{2}$

Stark ${ }^{16}$ reported a case of repeated dental implant failure due to etidronate. As the implants have no "epithelial anchorage" there is a greater tendency towards bone exposure.

\section{Conclusions}

All patients that are treated with bisphosphonates (especially intravenously) should be considered as being susceptible to osteonecrosis if they are subjected to invasive dental procedures, or maxillary or mandibular bone surgery. The key lies in proper planning before starting bisphosphonate treatment, as occurs with patients that are to undergo radiotherapy of the head and neck. ${ }^{2}$ Any dental problems that the patient may have in the short term should be anticipated and dealt with promptly so that the mucosa is intact once bisphosphonate therapy is started, and no bone is exposed.

Once osteonecrosis is established, a conservative approach should be adopted, as there is a possibility of iatrogenic worsening of the symptoms. The surgical procedures should also be conservative and they should be carried out only in the more symptomatic cases. 